\title{
Swiss Industrial Biocatalysis Consortium (SIBC)
}

\author{
Beat Wirz ${ }^{\star a}$, Matthias Kittelmann ${ }^{b}$, Hans-Peter Meyer ${ }^{c}$, and Roland Wohlgemuth ${ }^{\mathrm{d}}$
}

Dedicated to Armin Fiechter (1924-2010) - a beacon of biotechnology in Switzerland

\begin{abstract}
Taking up the common challenges in biocatalysis, a group of industrialists decided to react with a bottom-up solution, and created the Swiss Industrial Biocatalysis Consortium (SIBC). The Swiss Industrial Biocatalysis Consortium is a pre-competitive working group to better implement and utilize existing know-how and resources in biocatalysis, and to influence and shape the economic and educational political environment. Recent examples of activities are outlined.
\end{abstract}

Keywords: Biocatalysis · SIBC · Swiss Industrial Biocatalysis Consortium

\section{Introduction}

White or Industrial Biotechnology is an established tool for the synthesis of bulk and fine chemicals as well as pharmaceutical intermediates or flavors \& fragrances. ${ }^{[1]}$ The major technologies within White Biotechnology - fermentation and biocatalysis - have been key for many technical and economic success stories in the last decades. ${ }^{[2]}$ Particularly biocatalysis, which is defined as the one- or up to multi-step conversion of substrates to products by means of enzymes (enzymatic transformations), or whole cells (microbial transformations) is in many aspects methodologically closely related to chemical synthesis. ${ }^{[3]}$ Biocatalysis faces, however, a number of challenges in industrial large-scale applications. Time constraints to evaluate and establish a new reaction are among the most prominent, mainly because extensive optimization work is usually required to establish a technically viable process step for a novel, normally xenobiotic substrate. Building blocks, intermediates or metabolites for the pharmaceutical industry

\footnotetext{
${ }^{\star}$ Correspondence: Dr. B. Wirz ${ }^{\mathrm{a}}$

Tel.: + 41616883390

Fax: +41616881673

E-mail: beat.wirz@roche.com

aPharmaceuticals, Therapeutic Modalities

Process Research \& Synthesis Basel, Biocatalysis

F. Hoffmann-La Roche AG

$\mathrm{CH}-4070$ Basel

'GDC/PSB/Bioreactions

Novartis Pharma AG

$\mathrm{CH}-4002$ Basel

'Lonza AG

$\mathrm{CH}-3930$ Visp

${ }^{d}$ Research Specialties

Sigma-Aldrich

Industriestrasse 25

$\mathrm{CH}-9470$ Buchs
}

in particular have to be produced within short timelines, and the biocatalytic procedures are in competition with alternative technologies such as asymmetric chemical catalysis, classical chiral resolution of racemates, preparative chiral HPLC, SMB chromatography and an increasing number of building blocks from the chiral pool. The situation is very different with commodity chemicals and fine chemicals which have bulk character. Here production cost and economy of scale are the prime concerns while time constraints are much less of a topic, making a stringent optimization of the biocatalytic reaction necessary. ${ }^{[4]} \mathrm{De}-$ spite continuous progress, another challenge for industrial biocatalysis remains the still limited number of reaction types which can rely on a broad panel of commercial enzymes.

Because of these constraints and on the initiative of Hans-Peter Meyer (Lonza) a group of scientists representing the bio-

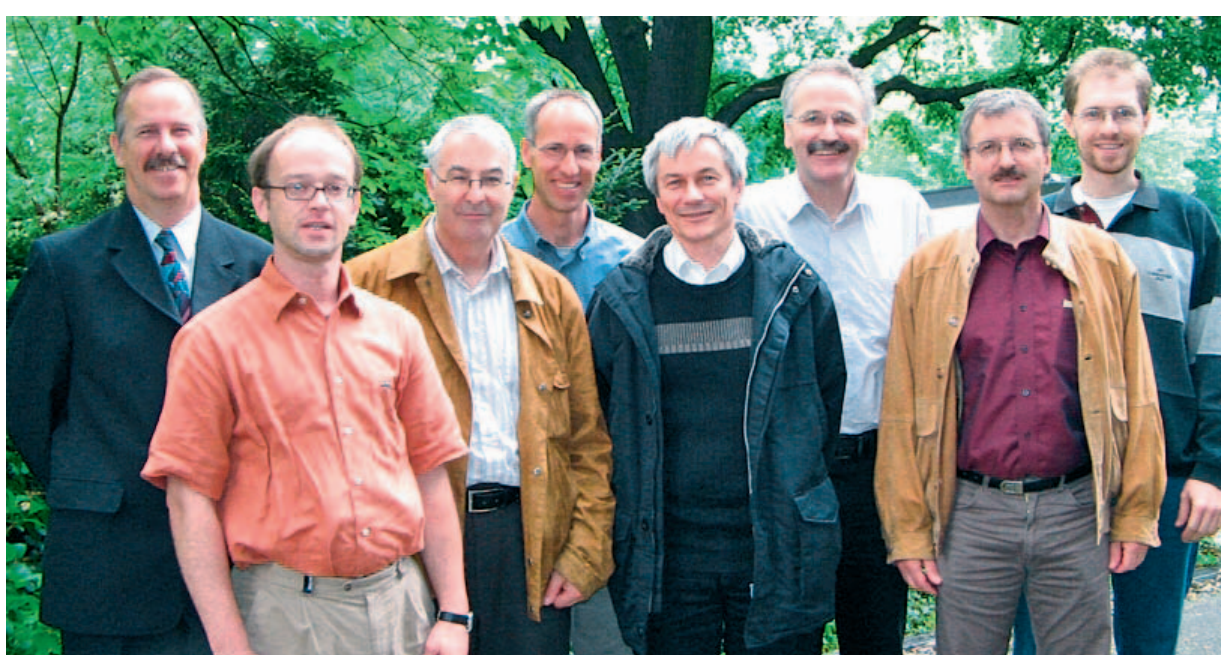

The 2004 founding team of the SIBC (from left): Thomas Keppler (Lonza), Franz Kaufmann (Ciba), Oreste Ghisalba (Novartis), Thomas Münch (Givaudan), Roland Wohlgemuth (Sigma-Aldrich), Hans-Peter Meyer (Lonza), Beat Wirz (Roche) and Volker Jungmann (Syngenta, heavily involved in the initial phase). catalysis platform in various Swiss industries ranging from the fine chemistry via pharmaceutical industry to flavors \& fragrances started to address these issues and founded the Swiss Industrial Biocatalysis Consortium (SIBC) in 2004. ${ }^{[5]}$ The idea behind this was to have a pre-competitive working group in order to better exploit existing know-how and resources in biocatalysis, and to influence and shape the economic and educational political environment. The major goal of the SIBC was explicitly to exchange not only information, but also living strains in order to improve the availability of characterized strains. This was necessary because of the far more diverse nature of biocatalytic processes as compared to therapeutic protein production or even secondary metabolite production. A broad enzyme toolbox is the key element for timely success. In addition, the SIBC was conceived as an industrial discussion and action platform 
for the mutual exchange of non-sensitive but relevant technical/scientific information. Furthermore, the SIBC has issued joint industrial statements in the field of biocatalysis regarding the needs of industrial biocatalysis, with the aim to establish a constructive dialogue between industrial and academic research. ${ }^{[6]}$

As mentioned above, the primary scope of the SIBC was to establish a joint microbial strain database by exchanging selected information on individual strain collections between SIBC members, creating a shared consortium strain database, to which each member contributes freely available data. Each contributing member, in return, has access to the collected information. The members were not only to exchange information, but also living strains in order to evaluate their usefulness for inhouse biotransformations under a respective Material Transfer Agreement. In the years to follow it turned out that the database has not yet been used as extensively as initially expected. One reason is assumed to be the absence of detailed structural data for the substrate molecules which could not be provided as they represent sensitive know-how to the companies. Moreover, a majority of the interesting strains were purchased by the member companies from international culture collections under an MTA and were therefore excluded from exchange.

The SIBC has also supported selected strategic activities, projects, congresses etc. in the field of biocatalysis or White Biotechnology, respectively. Examples are the consultancy support for the Culture Collection of Switzerland (CCOS) which was newly founded in 2009 by Prof. Martin Sievers or the organization of workshops at the last two 'Biotrans' conferences 2007 in Oviedo and 2009 in Bern (see below).

In addition, the SIBC is actively involved in formulating innovation areas of relevance for Switzerland and supporting policies in industrial biotechnology in close cooperation with the Swiss Biotech Association (SBA) and the Swiss Society of the Chemical Industry (SGCI).

The consortium members meet about three times a year to discuss current issues. Industrial membership has also undergone some changes since the start of the consortium. In 2009 CIBA left the SIBC as a consequence of the takeover by BASF. In May 2010, two new companies with activities and interest in industrial biocatalysis, DSM and Cerbios, have joined the SIBC. Contact details of all the members together with further information on the SIBC and some useful links can be found on http:// www.swissbiotech.org/ swiss_industrial_ biocatalysis consortium_sibc_.

The SIBC has always been keen to exchange intensively with other industries, academic and government institutions. Different platforms have been utilized to feel the pulse in industrial biotechnology. As mentioned above, workshops have been organized for this purpose by the SIBC at the 'Biotrans 2007' in Oviedo and at the 'Biotrans 2009' in Bern. As the detailed summaries of both workshops have been filed on the corresponding websites, the next section highlights only a few samples of statements and opinions expressed by different participants during the workshop last year. One or two of the statements below might put a smile on your face.

"Industry does not communicate relevant information and academia does not listen."

"Industry does not fund strategic research / technologies anymore but only specific product-oriented short-term research on products which are hot for the moment."

"Industry needs to consider and spend money on strategic R\&D as well."

"Big companies have a lack of knowledge on biocatalysis"

"Academia should concentrate on real problems, and stay away from solving problems which do not exist (However, there were comments that academia should not solve problems of industry but focus on enabling technologies)."

"Focusing appears to be demotivating for academia. Telling academia what should be done is a bad idea."

"The world is not aware of the huge potential biocatalyis or biotechnology can provide for a safer and greener world."

"What is lacking is the ability to define target molecules interesting later on."

"A large global consortium may be a good idea. Europe has already several consortia and clusters."

"The cost of enzyme is not a problem or a barrier. The problem is the availability of the raw materials (sugars etc.)."

The partly controversial discussion in the second workshop was much appreciated.

The experience of the two 'Biotrans' workshops with the excellent input from the global scientific community in biocatalysis has clearly shown the tremendous interest to move on to new horizons and to overcome scientific and technological, communicative and administrative as well as economic and commercial barriers.

It turns out that SIBC serves as an unusually pragmatic approach to common problems of different industries, which to our knowledge has no parallel elsewhere. The new global value creation chains clearly require adequate thoughts and actions on different organizational and geographical levels. The SIBC is a local industrial counterpart to scientific orga- nizations with initiatives like the vision 2025, the strategic research agenda and the corresponding implementation action plan, which have illustrated the necessary long-term commitment to biocatalysis and industrial biotechnology. ${ }^{[7]}$ The European Section on Applied Biocatalysis (ESAB), although founded in Europe, is open to any interested scientists worldwide and organizes highly successful conference series fostering the scientific and technological exchange without borders. Global organizations like the organization of economic cooperation and development (OECD) and UNIDO have become interested and recently also started activities in this area from a global perspective. ${ }^{[8]}$

In summary, it has been a great experience to see the SIBC develop from idea to reality, to build bridges across different industries in a small country and to overcome barriers. The future of industrial biocatalysis is now and the grand challenges require more fundamental innovation worldwide. With this view, the consortium is open to all companies having $R \& D$ activities in biocatalysis in Switzerland.

Received: September 6, 2010

[1] a) A. Schmid, J. S. Dordick, B. Hauer, A. Kiener, M. Wubbolts, B. Witholt, Nature 2001, 409, 258; b) A. J. Straathof, S. Panke, A. Schmid, Curr. Opin. Biotechnol. 2002, 13, 548; c) R. Wohlgemuth, New Biotechnol. 2009, 25, 204.

[2] a) 'Asymmetric Catalysis on Industrial Scale', Eds. H. U. Blaser, E. Schmidt, 2003, Wiley-VCH, Weinheim; b) 'Industrial Biotransformations', Eds. A. Liese, K. Seelbach, C. Wandrey, 2nd completely revised and extended edition, 2006, Wiley-VCH, Weinheim, c) W. L. Tang, H. Zhao, Biotechnol. J. 2009, 4, 1725; d) O. Ghisalba, H. P. Meyer, R. Wohlgemuth, 'Industrial Biotransformation', in 'Encyclopedia of Industrial Biotechnology', 2010, Ed. M. C. Flickinger, Wiley, Hoboken, NJ; e) 'Large-Scale Asymmetric Catalysis', Eds. H. U. Blaser, H. J. Federsel, 2010, Wiley$\mathrm{VCH}$, Weinheim.

[3] a) 'Modern Biocatalysis', Eds. W. D. Fessner, T. Anthonsen, 2009, Wiley-VCH, Weinheim; b) E. Garcia-Junceda, 'Multi-Step enzyme catalysis', 2008, Wiley-VCH, Weinheim.

[4] a) 'Biocatalysis for Green Chemistry and Chemical Process Development', Eds. J. Tao, R. J. Kazlauskas, 2010, Wiley, Hoboken, NJ; b) 'Industrial Biotechnology, Sustainable Growth and Economic Success', Eds. W. Soetaert, E. J. Vandamme, 2010, Wiley-VCH, Weinheim.

[5] Founding members were Ciba, Givaudan, Lonza, Novartis, Roche and Sigma-Aldrich.

[6] H. P. Meyer, T. Münch, Bioworld 2005, 1, 14.

[7] a) These documents can be found at http:// www.esabweb.org; b) M. Lex, Science Progress 2008, 91, 39

[8] OECD Working Party on Biotechnology, Outlook on Industrial Biotechnology, document presented at OECD workshop 13-15 January 2010, Vienna and available at the website http://www.olis.oecd.org/olis/2009doc.nsf/ ENGDATCORPLOOK/NTOOO089EA/\$FILE/ JT03276759.PDF 